\title{
Diferentes volumes de substrato no desenvolvimento de plantas de cravo-de-defunto (Tagetes patula 1. )
}

\author{
CHARLES ALLAN TELLES ${ }^{1}$; ÉRICA COSTA MIELKE²; MARÍLIA PEREIRA MACHADO ${ }^{2}$ e LUIZ ANTONIO BIASI ${ }^{3}$
}

\begin{abstract}
RESUMO
O objetivo deste trabalho foi avaliar o desenvolvimento de plantas de cravo-de-defunto (Tagetes patula L.) produzidas em diferentes volumes de substrato. O experimento foi realizado em casa-de-vegetação do Departamento de Fitotecnia e Fitossanitarismo da Universidade Federal do Paraná. O delineamento experimental utilizado foi inteiramente casualizado com quatro tratamentos, quatro repetições e dez plantas por parcela. Os volumes de substrato foram os seguintes: 47, 105,380 e $1000 \mathrm{~cm}^{3}$. Os efeitos dos tratamentos foram avaliados determinando-se as matérias fresca e seca da parte aérea e do sistema radicular, altura das plantas, número de botões e número de flores. Constatou-se que o desenvolvimento das plantas foi influenciado pelo volume de substrato. O maior volume de substrato apresentou os melhores resultados para todas as variáveis analisadas, porém o volume de $380 \mathrm{~cm}^{3}$ é o mais recomendado por conferir menor incremento ao custo final da produção, mantendo as características desejáveis para a comercialização.
\end{abstract}

Palavras-chave: produção de plantas, floríferas anuais, embalagem, Tagetes sp.

\section{ABSTRACT \\ Different volumes of growing medium in the development of plants of marigold (Tagetes patula L.)}

The objective of this work was to evaluate the development of marigold plants (Tagetes patula L.), produced in different volumes of growing medium.
The experiment was carried out in a greenhouse of the Department of Fitotecnia and Fitossanitarismo of UFPR. A completely randomized experimental design with 4 treatments, 4 replications and 10 plants per parcel, was used. The treatments had consisted of the following volumes of growing medium: 47, 105, 380 and $1000 \mathrm{~cm}^{3}$. The effect of the treatments had been evaluated determining the fresh and dry matter of the aerial part and the root system, height of the plants, number of buds and number of flowers. It was evidenced that the development of the plants was influenced by the volume of growing medium. The biggest volume presented the best results for all the analyzed variable. However the volume of 380 $\mathrm{cm}^{3}$ is recommended, for conferring minor increment to the final cost of the production, keeping the desirable characteristics for the commercialization.

Key words: plant production, ornamental bedding plant, packing, Tagetes sp.

\section{INTRODUÇÃO}

A produção de mudas e plantas de qualidade das culturas representa um dos mais importantes pré-requisitos para o sucesso do empreendimento agrícola, e o volume do substrato, segundo SILVA et al. (2001), é um dos critérios que devem ser considerados para a obtenção de mudas de boa qualidade.

Vários tamanhos de recipientes podem ser utilizados para a produção de mudas (CUNHA et al., 2002). Os volumes dos recipientes, e conseqüentemente de substrato, influenciam a disponibilidade de nutrientes e água (BÖHM, 1979). Em trabalhos realizados com cafeeiro, verificou-se que recipientes com maior volu-

\footnotetext{
${ }^{1}$ Engenheiro Agrônomo, Aluno do Programa de Pós-Graduação em Agronomia, Produção Vegetal, Universidade Federal do Paraná (UFPR), Bolsista da CAPES. Email: charles.allan@bol.com.br

${ }^{2}$ Engenheira Agrônoma, Aluna do Programa de Pós-Graduação em Agronomia, Produção Vegetal, UFPR.

3 Professor Dr. Adjunto do Departamento de Fitotecnia e Fitossanitarismo, Setor de Ciências Agrárias, UFPR. Caixa Postal 19.061, 81531-990 Curitiba (PR). Email: biasi@ufpr.br. Bolsista de Produtividade em Pesquisa do CNPq.
} 
me de substrato apresentam uma tendência a produzir mudas mais vigorosas e de melhor qualidade (GODOY \& GODOY JR., 1965; SILVEIRA et al., 1973; BESAGOITIA, 1980).

Além de exercer influência sobre a qualidade das mudas, o volume de substrato pode se apresentar como um grande diferencial no custo da produção, pois o substrato representa de 8 a $10 \%$ desse custo (FNP, 2001). Portanto, é fundamental determinar, com a maior precisão possível, o volume de substrato para que a produção se torne viável.

Diversos autores demonstraram que a utilização de maior ou menor quantidade de substrato interfere no desenvolvimento de mudas de diferentes espécies e no florescimento e frutificação das plantas (JESUS et al., 1987; NIMURA et al., 1991; MELO \& MENDES, 2000; SIRIN et al., 1999; HEALY et al., 1993).

A espécie Tagetes patula L., conhecida como cravo-de-defunto, é uma planta ornamental de ciclo rápido, que apresenta flores em tonalidade variável de amarelo, alaranjado e marrom-avermelhado, reunidas em capítulos densos, o que a torna uma planta muito apreciada para ornamentação de jardins com rápidas substituições da composição florística. Além disso, é uma espécie de ciclo anual que pode ser cultivada, com sucesso, em regiões tropicais durante o período do verão (LORENZI \& SOUZA, 1999).

O objetivo deste trabalho foi avaliar o desenvolvimento de plantas de cravo-de-defunto produzidas em diferentes volumes de substrato.

\section{MATERIAIS E MÉTODOS}

O experimento foi realizado na casa-de-vegetação do Departamento de Fitotecnia e Fitossanitarismo do Setor de Ciências Agrárias da Universidade Federal do Paraná, em Curitiba -PR, no período de outubro a novembro de 2003.

A planta ornamental utilizada foi o cravo-de-defunto; as mudas foram adquiridas de um produtor da região, produzidas em bandejas com 250 células em substrato vermiculita. No momento da instalação do experimento, as mudas apresentavam uma altura média de $4,5 \mathrm{~cm}$, com 2 folhas de $3,5 \mathrm{~cm}$ de comprimento, 2 folhas de $1,0 \mathrm{~cm}$ (compostas de 5 folíolos) e 2 folhas de $2,0 \mathrm{~cm}$ (folha com um único folíolo).

Os tratamentos foram embalagens com diferentes volumes de substrato: $47 \mathrm{~cm}^{3}, 105 \mathrm{~cm}^{3}, 380 \mathrm{~cm}^{3} \mathrm{e}$ $1000 \mathrm{~cm}^{3}$. O substrato utilizado foi da marca comercial Plantmax $^{\circledR}$, com as seguintes características químicas: $\mathrm{pH} \mathrm{SMP}=6,0 ; \mathrm{pH} \mathrm{CaCl}_{2}=5,0 ; \mathrm{Al}^{+3} \mathrm{cmol}_{\mathrm{c}} / \mathrm{dm}^{3}=0,2$;
$\mathrm{H}^{+}+\mathrm{Al}^{+3} \mathrm{cmol}_{\mathrm{c}} / \mathrm{dm}^{3}=5,0 ; \mathrm{Ca}^{+2} \mathrm{cmol}_{\mathrm{c}} / \mathrm{dm}^{3}=15,45 ;$ $\mathrm{Ca}^{+2}+\mathrm{Mg}^{+2} \mathrm{cmol}_{\mathrm{c}} / \mathrm{dm}^{3}=23,88 ; \mathrm{K}^{+} \mathrm{cmol}_{\mathrm{c}} / \mathrm{dm}^{3}=1,92 ;$ $\mathrm{T} \mathrm{cmol} / \mathrm{dm}^{3}=30,8 ; \mathrm{P} \mathrm{mg} / \mathrm{dm}^{3}=492,8 ; \mathrm{C} \mathrm{g} / \mathrm{dm}^{3}=108,5$ e $\mathrm{V} \%=83,77$. As características físicas, determinadas pela metodologia de FRETZ et al. (1979), foram as seguintes: densidade $=495,7$ g. $\mathrm{L}^{-1}$; espaço poroso total $(\mathrm{EPT})=51,5 \%$; água retida na capacidade de campo $($ ARCC $)=34,8 \%$ e espaço de ar na capacidade de campo $($ EACC $)=16,7 \%$.

As embalagens utilizadas foram sacos de plástico preto, tendo sido mantida a altura de $15 \mathrm{~cm}$ para todos os tratamentos, variando apenas o diâmetro das embalagens. Para conseguir o diâmetro desejado, os sacos foram grampeados com grampeador comum.

A umidade do substrato foi mantida em sua capacidade de campo para os diferentes tratamentos com irrigação diária, irrigando-se até completa saturação (até escorrimento da água livre por baixo do recipiente), principalmente para a embalagem de menor diâmetro, que apresentava uma desidratação mais rápida.

No decorrer do experimento, não foi efetuada nenhuma adubação no substrato devido ao fato de ele se apresentar bastante fértil e de a planta ser de ciclo rápido, indo rapidamente a campo.

O delineamento experimental utilizado foi inteiramente ao acaso com 4 repetições e 10 plantas por parcela, totalizando 160 plantas no experimento.

A distribuição das plantas sobre a bancada da estufa foi realizada de maneira homogênea, de modo que nenhum tratamento levasse vantagem sobre o outro com relação a recebimento de luz e irrigação.

O experimento foi avaliado após 43 dias de instalação, observando-se as seguintes variáveis: altura da planta (medida do colo até a folha mais alta), número de botões florais fechados, número de flores abertas, massas fresca e seca da parte aérea e sistema radicular.

Para determinação da altura da planta foi utilizada uma régua graduada em milímetros e para a massa foi utilizada balança de precisão em miligramas. Antes de a massa das raízes ser medida, elas foram lavadas em água corrente para retirar todo o substrato; a fim de eliminar o excesso de umidade, as raízes secaram à sombra. Para medir a massa seca, as plantas (parte aérea e parte radicular) foram colocadas para secar em estufa de $65^{\circ} \mathrm{C}$ por um período de 64 horas.

Para a análise estatística utilizou-se o programa estatístico Stati Graphics 5. Para determinar a diferença entre os tratamentos, as médias foram submetidas ao teste de Tukey com $\mathrm{p}>0,05$. 


\section{RESULTADOS E DISCUSSÃO}

O cravo-de-defunto apresentou seu crescimento limitado de acordo com o volume de substrato utilizado (FIGURA 1), sendo possível inferir que a cultura apresenta certa exigência na quantidade de substrato, podendo esses resultados ser extrapolados para outras plantas ornamentais de ciclo anual que apresentam características semelhantes.

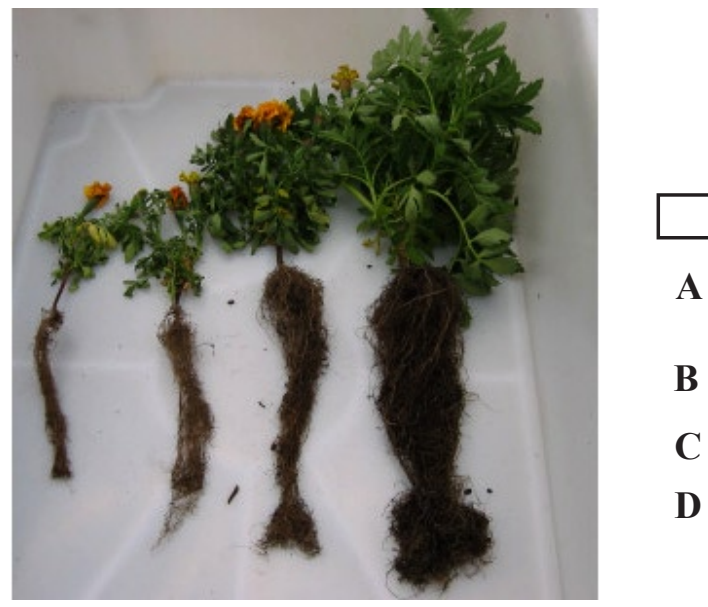

Figura 1. Desenvolvimento das mudas de cravo-dedefunto em diferentes volumes de substrato $47 \mathrm{~cm}^{3}$ (A), $105 \mathrm{~cm}^{3}$ (B), $380 \mathrm{~cm}^{3}$ (C) e $1000 \mathrm{~cm}^{3}$ (D)

As plantas produzidas no maior volume de substrato apresentaram maior massa fresca e seca da parte aérea. Os dois menores volumes de substrato restringiram o desenvolvimento da parte aérea das plantas, que apresentaram menor massa fresca e seca (Tabela 2); isso mostra que o volume de substrato influenciou a disponibilidade de nutrientes e água, como citado por BÖHM (1979).
Já para a massa do sistema radicular, o tratamento de maior volume $\left(1000 \mathrm{~cm}^{3}\right)$ apresentou-se superior aos demais, uma vez que a massa seca foi de 1,04 grama e a massa fresca 2,69 gramas. Esse fato foi observado devido às raízes terem à sua disposição uma maior área de crescimento e exploração. Outro fator que poderia explicar esses resultados é que o substrato em recipientes de maior volume apresentavam um volume de macroporos maior (Tabela 1), que, segundo KÄMPF (2000), beneficia a respiração das raízes, pois permite uma remoção mais rápida do gás carbônico formado e eleva o suprimento de oxigênio, permitindo uma boa troca gasosa e um melhor crescimento e desenvolvimento radicular.

As plantas também apresentaram uma área foliar maior, conforme o aumento do volume de substrato (Figura 1), o que implicou uma maior taxa de fotossíntese, favorecendo a produção de açúcares que ajudaram no crescimento radicular, assim como encontrado por NIMURA et al. (1991), que constataram em maiores recipientes aumentos do número de folhas, de área foliar, de ramos, de massa fresca e seca de raiz em Dieffenbachia sp.

Esses resultados quanto à área foliar são considerados muito bons, pois quanto maior a área foliar e sua altura, a planta tem maior capacidade e mais regiões para emissão de botões florais, o que é desejado. Esse fato pode também estar relacionado à capacidade de retenção hídrica (Tabela 1), pois, conforme se aumenta o volume de substrato, há um maior volume de água retida na capacidade de campo, permitindo uma maior disponibilidade de água para planta, e um maior volume de ar na capacidade de campo, permitindo que a plantas se desenvolvam de maneira satisfatória.

Tabela 1. Características físicas do substrato Plantmax ${ }^{\circledR}$, determinada de acordo com metodologia de FRETZ et al. (1979), com relação ao espaço poroso total (EPT), água retida na capacidade de campo (ARCC) e espaço de ar na capacidade campo (EACC), nos diferentes volumes estudados do substrato

\begin{tabular}{|l|r|r|r|}
\hline Volume & EPT & ARCC & EACC \\
\hline \multicolumn{4}{|c|}{$\mathrm{cm}^{3}$} \\
\hline 47 & 24,2 & 16,3 & 7,8 \\
\hline 105 & 54,1 & 36,5 & 17,5 \\
\hline 380 & 195,7 & 132,2 & 63,4 \\
\hline 1000 & 515,0 & 348,0 & 167,0 \\
\hline
\end{tabular}


Tabela 2. Massa seca e fresca da parte aérea e radicular, número de botões emitidos, número de flores abertas e altura das plantas de cravo-de-defunto em diferentes volumes de substrato Plantmax ${ }^{\circledR}$, após 43 dias de cultivo

\begin{tabular}{|l|c|c|c|c|c|c|c|}
\hline \multirow{2}{*}{ Tratamento } & $\begin{array}{c}\text { Massa seca } \\
\text { parte aérea }\end{array}$ & \multicolumn{1}{|c|}{$\begin{array}{c}\text { Massa fresca } \\
\text { parte aérea }\end{array}$} & $\begin{array}{c}\text { Massa seca } \\
\text { sist. radicular }\end{array}$ & $\begin{array}{c}\text { Massa fresca } \\
\text { sist. radicular }\end{array}$ & Botões/planta & Flores/ planta & Altura \\
\cline { 2 - 9 } & \multicolumn{9}{|c|}{ gramas } & \multicolumn{4}{|c|}{$\mathrm{n}^{\mathrm{o}}$} & \multicolumn{2}{c|}{$\mathrm{cm}$} \\
\hline $47 \mathrm{~cm}^{3}$ & $0,65 \mathrm{c}$ & $5,28 \mathrm{c}$ & $0,17 \mathrm{~b}$ & $0,26 \mathrm{~b}$ & $1,42 \mathrm{c}$ & $1,47 \mathrm{~b}$ & $11,67 \mathrm{c}$ \\
\hline $105 \mathrm{~cm}^{3}$ & $0,91 \mathrm{c}$ & $5,91 \mathrm{c}$ & $0,27 \mathrm{~b}$ & $0,45 \mathrm{~b}$ & $1,72 \mathrm{c}$ & $1,97 \mathrm{~b}$ & $13,01 \mathrm{c}$ \\
\hline $380 \mathrm{~cm}^{3}$ & $2,49 \mathrm{~b}$ & $15,57 \mathrm{~b}$ & $0,39 \mathrm{~b}$ & $0,59 \mathrm{~b}$ & $3,12 \mathrm{~b}$ & $3,4 \mathrm{a}$ & $15,96 \mathrm{~b}$ \\
\hline $1000 \mathrm{~cm}^{3}$ & $4,28 \mathrm{a}$ & $36,40 \mathrm{a}$ & $1,04 \mathrm{a}$ & $2,69 \mathrm{a}$ & $4,92 \mathrm{a}$ & $3,5 \mathrm{a}$ & $22,58 \mathrm{a}$ \\
\hline C.V. $\%$ & 17,85 & 6,73 & 26,08 & 22,39 & 14,47 & 14,09 & 8,64 \\
\hline
\end{tabular}

Médias seguidas por letras distintas diferem entre si ao nível de significância de $5 \%$ pelo teste de Tukey.

Quanto ao número de botões e altura, houve diferença significativa entre os tratamentos, exceto entre os dois volumes de substrato menores, que se apresentaram iguais estatisticamente (Tabela 2).

Analisando o número de flores, os volumes 380 e $1000 \mathrm{~cm}^{3}$ apresentaram-se superiores comparativamente com os de menor volume, não havendo diferença estatística entre eles.

Esses resultados demonstram que o volume de substrato afeta diretamente o desenvolvimento e florescimento de plantas cultivadas em recipientes, como já verificado em trabalhos em que se comprovou a interferência do volume de substrato no desenvolvimento, florescimento e frutificação das plantas (JESUS et al., 1987; NIMURA et al., 1991; MELO \& MENDES, 2000; SIRIN et al., 1999; HEALY et al., 1993). JESUS et al. (1987) observaram uma grande influência do tamanho do recipiente e, conseqüentemente, do volume de substrato na altura de plantas de louro (Cordia trichotoma) e gonçalo-alves (Astronium fraxinifolium). Estudando plantas denominadas forrações, IERSEL (1997) verificou que as plantas alocadas em volume maior de substrato tiveram crescimento acelerado devido à maior taxa de área foliar e, em situação contrária, ramos laterais e expansão foliar foram suprimidos pela restrição radicular e a floração foi atrasada.

No presente trabalho não foi constatado atraso no início do aparecimento do primeiro botão, e sim na quantidade e na qualidade dos mesmos. Isso pode estar relacionado ao crescimento do sistema radicular e ao fornecimento de nutrientes, pois, apesar do substrato Plantmax ${ }^{\circledR}$ apresentar bons níveis de nutrientes, o volume cedido a cada tratamento limitou o seu fornecimento.

Embora esse comportamento seja esperado, comercialmente o volume de $380 \mathrm{~cm}^{3}$ já seria compatível com o que é considerado pelos floricultores como padrão ideal, ou seja, pelo menos duas flores abertas e de 2 a 3 botões formados. Considerando os custos entre os dois melhores tratamentos, há uma diferença de $263 \%$ no valor despendido apenas em substrato.

Embora as plantas cultivadas no maior volume de substrato tenham apresentado melhor desenvolvimento, o volume intermediário de 380 $\mathrm{cm}^{3}$ é o mais recomendado por apresentar um menor incremento no custo final da produção, mantendo as características desejáveis para a comercialização.

\section{CONCLUSÃO}

$\mathrm{O}$ volume de substrato afeta o desenvolvimento das mudas de cravo-de-defunto devido, principalmente, ao suprimento de água, nutrientes e espaço para o crescimento.

O volume de $380 \mathrm{~cm}^{3}$ mostrou-se satisfatório na produção de mudas de cravo-de-defunto, pois as mesmas apresentaram o padrão desejado pelo floricultor.

\section{REFERÊNCIAS BIBLIOGRÁFICAS}

BESAGOITIA, M.C.R. Efecto del tamanõ de la bolsa en el desarrolo del cafetos cultivares 'Bourbon' y 'pacas' en vivero. Resúmenes de Investigaciones en café - 1979/ 1980, Nueva San Salvador, v. 3, p. 71-72, 1980.

BÖHM, W. Methods of studying root systems. Berlin: SpringerVeriag, 1979. 188p.

CUNHA, R.L. da; SOUZA, C.A.S.; NETO, A.A.; MELO, B. de \& CORRÊA, J.F. Avaliação de substratos e tamanhos de recipientes na formação de mudas de cafeeiro (Coffea arabica L.) em tubetes. Ciência e Agrotecnologia, Lavras, v. 26, n. 1, p.7-12, 2002. 
FNP Consultoria \& Comércio. Perspectiva do mercado de flores e plantas ornamentais no Brasil. In: Agrianual 2001 - Anuário da Agricultura Brasileira. São Paulo: Editora Argos, 2001. p.337-349.

FRETZ, T.A.; READ, P.E. \& PEELE, M.C. Plant propagation Lab. Manual Burgess Publishiny Company, Minneapolis, USA, p.15-19, 1979.

GODOY, O.P. \& GODOY JÚNIOR, C. Influência da adubação no desenvolvimento de mudas de café. Revista de Agricultura, Piracicaba, v. 40, n. 3, p. 125-129, 1965.

HEALY,W.; KLICK, S.; ROH, M.S. \& LAWSON, R.H. Potting method improved flowering of alstroemeria. Acta Horticulturae, Baltimore, n. 337, p.19-24, 1993.

IERSEL, V.M. Root restriction effects on growth and development of salvia (Salvia splendens). HortScience, Georgia, v.32, n.7, p.1186-1190, 1997.

JESUS, R.M. de; MENANDRO, M. de S; BATISTA, J.L.F; COUTO, H.T.Z. de; JESUS, R.M. \& COUTO, do H.T.Z. Effect of container size, type of substrate and shading on seedling growth of louro (Cordia trichotoma) and goncalo-alves (Astronium fraxinifolium).IPEF, Instituto de Pesquisa e Estudos Florestais, n.37, p. 13-19, 1987.

KAMPF, A.N. Produção comercial de plantas ornamentais. Guaíba: Agropecuária, 2000, 254p.
LORENZI, H. \& SOUZA, H.M. Plantas ornamentais do Brasil: arbustivas, herbáceas e trepadeiras.2. ed. Nova Odessa: Editora Plantarum. 1999. 1088p.

MELO, B. de \& MENDES, A.N.G. Container sizes and plant development at several stages of seedling production in coffe (Coffea arabica L.). Ciência e Agrotecnologia, Lavras, v.24, n.3, p. 679-687, 2000.

NIMURA, M.; MORIOKA, K.; HARA, M.; NISHINO, J. \& YONEMURA, K. Influences of pot spacing, pot size and the number of transplanting on growth and plant quality in Dieffenbachia. Bulletin of the Aichi-ken Agricultural Research Center, n. 23, p.227-236, 1991.

SILVA, R.P. da.; PEIXOTO, J.R. \& JUNQUEIRA, N.T.V. Influência de diversos substratos no desenvolvimento de mudas de maracujá-azedo (Passiflora edulis Sims f. flavicarpa Deg.). Revista Brasileira de Fruticultura, Cruz das Almas, v.23, n.2, p.337-381, 2001.

SILVEIRA, A.J. da; SANTANA, D.P. \& PEREIRA, M.L. Efeito do tamanho do saco plástico e do método de semeadura no desenvolvimento de mudas de café. Seiva, Viçosa, v. 33, n. 77, p. 8-14, 1973.

SIRIN, U.; SEVGICAN, A.; TUZEL,Y.; BURRAGE, S.W.; BAILEY, B.J.; GUL,A.; SMITH, A.R. \& TUNCAY, O. The effect of pot size and growing media of tomato in soiless culture. Acta Horticulturae, Antalya, n.491, p. 343-348, 1999. 